Old Dominion University

ODU Digital Commons

Physics Faculty Publications

Physics

$3-2013$

\title{
Experimental Investigation of Long-Lived Rydberg States in Ultracold Argon
}

G. Ranjit

Old Dominion University

C. I. Sukenik

Old Dominion University, csukenik@odu.edu

Follow this and additional works at: https://digitalcommons.odu.edu/physics_fac_pubs

Part of the Atomic, Molecular and Optical Physics Commons

\section{Repository Citation}

Ranjit, G. and Sukenik, C. I., "Experimental Investigation of Long-Lived Rydberg States in Ultracold Argon" (2013). Physics Faculty Publications. 3.

https://digitalcommons.odu.edu/physics_fac_pubs/3

\section{Original Publication Citation}

Ranjit, G., \& Sukenik, C.I. (2013). Experimental investigation of long-lived Rydberg states in ultracold argon. Physical Review A, 87(3), 033418. doi: 10.1103/PhysRevA.87.033418 


\title{
Experimental investigation of long-lived Rydberg states in ultracold argon
}

\author{
G. Ranjit and C. I. Sukenik \\ Department of Physics, Old Dominion University, Norfolk, Virginia 23529, USA
}

(Received 1 October 2012; published 25 March 2013)

\begin{abstract}
We report on our investigation of the formation and survival of long-lived Rydberg states in argon produced by pulsed laser excitation of ultracold metastable state argon atoms in a magneto-optical trap. The states studied have a ${ }^{2} P_{1 / 2}$ core. Low angular momentum Rydberg states with this core normally autoionize rapidly. If, however, atoms are excited in the presence of electric fields, higher angular momentum states, traditionally termed "ZEKE states" (ZEKE is derived from "zero kinetic energy") can be formed. The lifetime of these states can be orders of magnitude greater than low angular momentum states. In this paper, we report on the time dependence of ZEKE Rydberg state population in an ultracold environment.
\end{abstract}

DOI: 10.1103/PhysRevA.87.033418

PACS number(s): 32.80.Ee, 33.80.Rv, 32.70.Cs

\section{INTRODUCTION}

There is considerable interest in the dynamics of ultracold plasmas and ultracold Rydberg gases. Such plasmas reside in or near the strong-coupling regime and, therefore, can serve as laboratory models for studying physical systems - such as white dwarfs - that would otherwise be difficult to explore. Ultracold plasmas are typically formed by photoexcitation of ultracold atoms to an energy region near (both above and below) an ionization threshold [1]. Excitation to bound highly excited Rydberg states can lead to the formation of a plasma via several processes, including collisions between Rydberg atoms. Conversely, Rydberg atoms can be formed by three-body recombination in an ultracold plasma [2-4]. Understanding the dynamics of ultracold Rydberg gases is, therefore, important for understanding the dynamics of ultracold plasmas.

We are interested in a particular class of ultracold Rydberg atoms, known, for historical reasons, as zero kinetic-energy (ZEKE) state Rydberg atoms. Because these Rydberg states were discovered in ZEKE photoelectron spectroscopy experiments being conducted to precisely determine ionization limits, they became known as ZEKE Rydberg states [5-7]. ZEKE Rydberg states are high angular momentum $(l$ and $m$ ) excited states, typically found in a region a few tens of wave numbers below an ionization threshold, that can be formed by laser excitation in the presence of electric fields (applied or stray) that enable $l$ and $m$ mixing during excitation. A uniform electric field mixes $l$ states, the atomic states become Stark states, and $l$ is no longer a good quantum number. If the field configuration does not possess cylindrical symmetry, then $m$ states can also be mixed.

In the case studied here, involving excitation of argon (Ar) atoms to a region just below the ${ }^{2} P_{1 / 2}$ (core) ionization threshold, the absence of electric fields during excitation would mean that high angular momentum states would be optically dark. Only low angular momentum states that decay very quickly (picosecond-to-nanosecond time scale) by radiationless autoionization through a core spin flip due to the Rydberg electron-core interaction would be accessible. These autoionizing states (primarily $s^{\prime}, p^{\prime}$, and $d^{\prime}$ states) in Ar and other noble gases have been studied extensively over many years, primarily through single- and multiphoton highresolution spectroscopy where the linewidth of the observed resonances can be used to extract the lifetime of the states [8-15]. Experimental studies of these low- $l$ states have been compared to theoretical calculations to better understand the dynamics of autoionization [16-20]. For a recent detailed review, see Ref. [21]. Whereas, the vast majority of spectroscopic measurements involve low- $l$ states, recently, optical spectroscopy for high- $l$ states with principal quantum $n$ in the range of $n=9-17$ [22] and high-precision microwave spectroscopy for states with $n=10, l>5$ [23] have also been reported for argon. In those studies, the focus was on structure measurements to extract dipole polarizabilities and quadrupole moments.

For very high angular momentum ZEKE states, the interaction of the Rydberg electron with the core is significantly reduced. Hence, ZEKE Rydberg state lifetimes are greatly enhanced compared to low angular momentum states with nearly a 2 orders of magnitude increase in lifetime reported [24-29]. Calculations were found to be in qualitative agreement with these results but often underestimated the ZEKE state survival if only $l$ mixing, but non- $m$ mixing, was included [30-33]. In addition, although the radiative lifetime scales as $n^{3}$ for low- $l$ states, this same $n$ scaling does not hold for ZEKE states. Calculations for hydrogen, for example, show that the lifetime scales to good approximation as $n^{5}$ for the highest- $l$ states and that the average lifetime for a statistical mixture of $l m$ states of the same $n$ manifold scales as $n^{4.5}$ [34]. Most experiments involving ZEKE states have been conducted at or near room temperature and with molecules. Several studies did investigate atoms - including argon [26,31] and xenon [28], and the formation of high- $l$ states has also been observed in an ultracold Rydberg gas of rubidium [35].

In this paper, we report on our investigation of the formation and survival of ZEKE Rydberg states in ultracold argon in an energy region just below the ionization threshold of the ${ }^{2} P_{1 / 2}$ ionic core. This threshold is $1431.58 \mathrm{~cm}^{-1}$ above the ionization threshold of the ${ }^{2} P_{3 / 2}$ ionic core Rydberg series [20]. Two tunable pulsed dye lasers are used to excite $\mathrm{Ar}^{*}$ atoms in a magneto-optical trap (MOT) to Rydberg states. After a variable delay, these atoms are ionized by applying an electric-field pulse, and the subsequent ions are detected and counted, allowing us to study the time dependence of the ZEKE Rydberg state population over a wide range of $n$ levels. This work represents an important study of noble-gas ZEKE Rydberg states interacting in an ultracold environment. 


\section{EXPERIMENTAL SETUP}

The experiment begins in a noble-gas magneto-optical trap that has been described previously [36]. Typically, the MOT contains $\sim 10^{6}$ atoms in the $3 p^{5}\left({ }^{2} P_{3 / 2}\right) 4 s\left(3 p^{5} 4 s[3 / 2]_{2}\right)$ metastable state and with a nearly Gaussian spatial distribution with a $\sim 1-\mathrm{mm}$ diameter. The trapping transition to the $3 p^{5} 4 p[5 / 2]_{3}$ state takes place at $811 \mathrm{~nm}$. Data are collected at $10 \mathrm{~Hz}$, corresponding to the repetition rate of the Nd:YAG (yttrium aluminum garnet) laser (Quantel Brilliant) used to pump two pulsed dye lasers. The flashlamp sync of the YAG laser (defined as $t=0$ ) is used to trigger a pulse generator (Quantum Composers, Inc.) that controls all subsequent timing of the experiment. At $t=0$, acousto-optic modulators are used to shutter the trapping and Zeeman slower laser light. The $Q$ switch for the laser fires at $t=186 \mu \mathrm{s}$, pumping two 5-ns pulsed dye lasers. The first is a homebuilt laser, pumped by 532-nm light and operating at $706 \mathrm{~nm}$ that produces $100-200 \mu \mathrm{J} /$ pulse. This laser is resonant with the $3 p^{5} 4 s[3 / 2]_{2} \rightarrow 3 p^{5} 4 p^{\prime}[3 / 2]_{2}$ transition at $706 \mathrm{~nm}$. (We use primed and unprimed terms to represent the terms generated from ${ }^{2} P_{1 / 2}$ and ${ }^{2} P_{3 / 2}$, respectively). The second is a Quantel TDL60, pumped by 355-nm light and tunable around $470 \mathrm{~nm}$, producing $\sim 1.5 \mathrm{~mJ} /$ pulse, that further excites atoms to highlying Rydberg levels near the ionization limit (see Fig. 1). In the absence of an electric field, this laser will excite $n s^{\prime}$ and $n d^{\prime}$ autoionizing states only. Finally, after a variable delay time, a voltage with a slew rate and amplitude of $50.24 \mathrm{~V} / \mu$ s and $-62.8 \mathrm{~V}$ is applied to one of two thin metal rings surrounding the MOT (the second ring is grounded) in order to field ionize the Rydberg atoms. Ions are, subsequently, detected

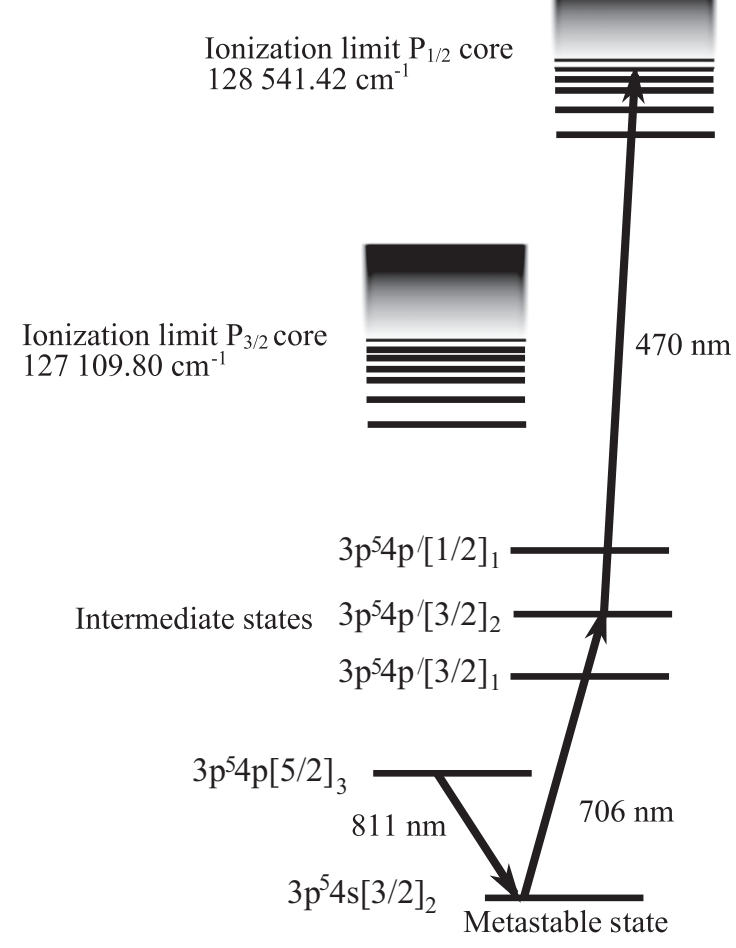

FIG. 1. Two-color two-photon excitation scheme for the production of Rydberg states of argon.

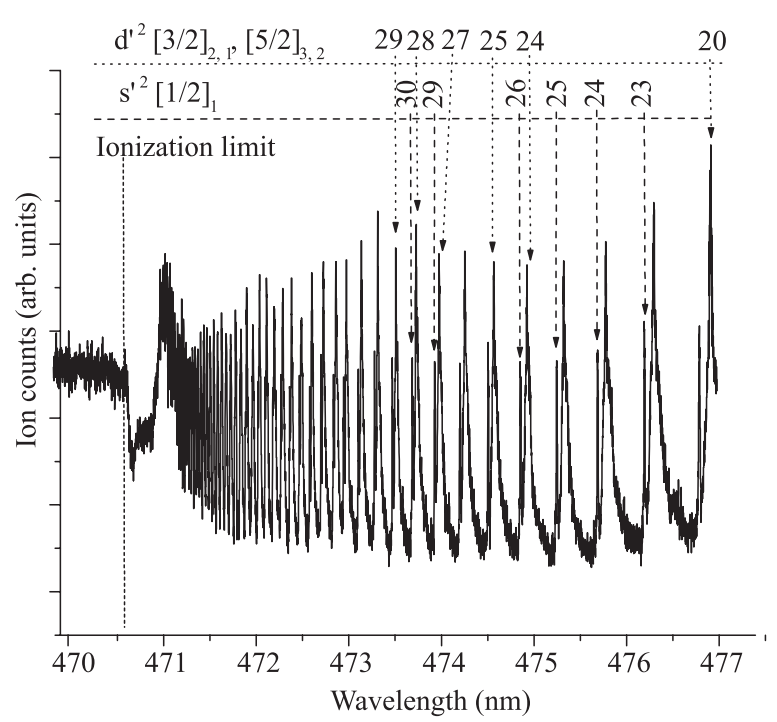

FIG. 2. The Rydberg spectrum of ${ }^{40} \mathrm{Ar}$ obtained using a two-color two-photon excitation scheme: $3 p^{5} 4 s[3 / 2]_{2} \rightarrow 3 p^{5} 4 p^{\prime}[3 / 2]_{2} \rightarrow$ Rydberg state, using $\sim 706$ - and $\sim 470$-nm light as the first and second excitation steps, respectively.

in a channel electron multiplier (CEM) and are counted on a multichannel scalar (Stanford Research Systems SR430).

\section{RESULTS AND DISCUSSION}

The spectrum of Rydberg levels under investigation in our study is shown in Fig. 2. Here, the first laser is fixed at $706 \mathrm{~nm}$, whereas, the second laser scans from 470 to $477 \mathrm{~nm}$. To obtain the spectrum, a pulsed electric field is applied $1 \mu$ s after photoexcitation. Ions created through pulsed field ionization (PFI) along with those created by autoionization and resonantly enhanced multiphoton ionization (REMPI) are swept to the CEM. Both $s^{\prime}$ and $d^{\prime}$ states are visible in the spectrum. With our laser power and bandwidth, individual Rydberg levels are resolved up to $n=65$.

The survival of ZEKE Rydberg states was measured by counting the number of field-ionized atoms as a function of delay time after photoexcitation of atoms with no voltage applied to the rings surrounding the MOT during laser excitation. Figure 3 shows a typical time sequence of subsequent ion detection. The multichannel scalar is triggered with a delay of $180 \mu$ s after the flashlamp of the Nd-YAG laser. Here, the dye laser, with an energy of $1.35 \mathrm{~mJ} /$ pulse, is parked so as to excite atoms to Rydberg states around $n=166$. Three features are visible in the figure. The first spike is a pick up of electrical noise from the laser $Q$ switch that conveniently marks the arrival of the 5-ns dye laser pulses. At $\sim 27 \mu$ s, after the atoms are excited, ions created through autoionization and REMPI arrive at the detector. Finally, ions created through PFI of the ZEKE states when the ramped voltage is initiated at $96 \mu \mathrm{s}$ after Rydberg excitation are detected.

The arrival time of the autoionized ions suggests the presence of a stray electric field, likely originating from the CEM field that penetrates through a grounded mesh in front of the detector. The energy gained by the ion via autoionization is a small fraction of the $\sim 1431.58 \mathrm{~cm}^{-1}$ available because most of the energy goes to the much lighter electron. The energy 


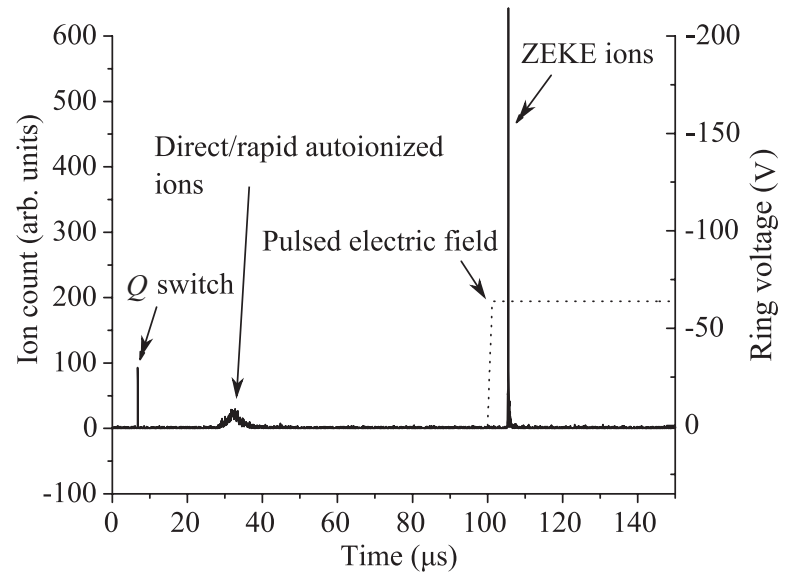

FIG. 3. Observed ion signals due to direct and autoionization and field-ionized ZEKE Rydberg atoms at an energy level corresponding to $n \sim 166$. The pulsed electric field is applied $94 \mu$ s after laser excitation.

gained results in an ion kinetic energy that would predict an arrival time of $\sim 10 \mathrm{~ms}$ after the laser pulse. By applying a small bias voltage to the ring closest to the CEM, we, indeed, observed an arrival time shift and eventual elimination of the ion signal, consistent with the presence of a stray electric field.

The number of ZEKE states in the MOT region at any one time will depend on both the number created initially and how long they survive. For example, in Fig. 4, we show the number of ZEKE Rydberg atoms detected as a function of laser wavelength for a fixed PFI delay time of $50 \mu \mathrm{s}$. This result is superimposed on the spectra of ions created solely by the laser without the application of an external ionizing field pulse where the photon counter gate opens at $1 \mu \mathrm{s}$ after the laser pulse. Below $n \sim 75$, very few or no ZEKE state ions are collected after the delay of $50 \mu \mathrm{s}$, even though the pulsed electric field is sufficient to ionize even red Stark states states

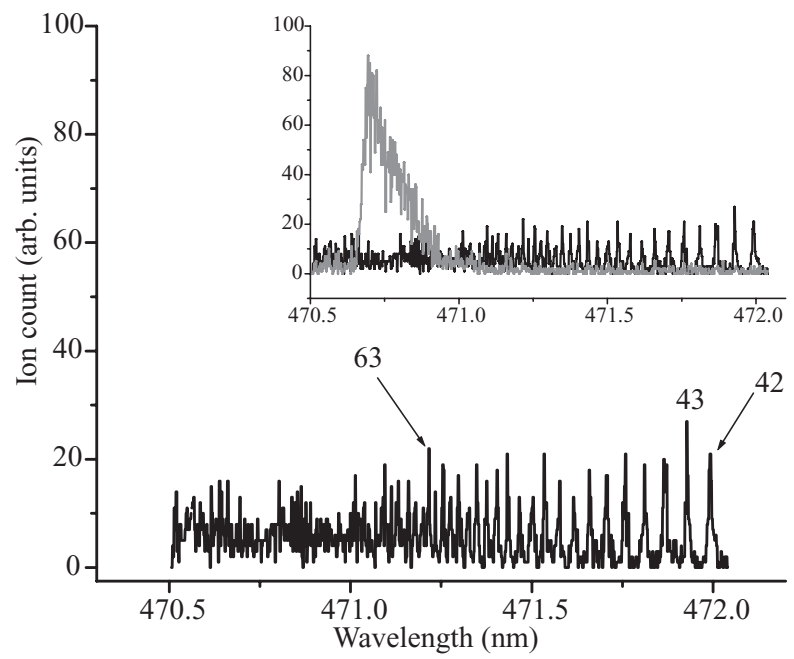

FIG. 4. Observed ions collected in a 40- $\mu$ s-wide gate that opens $1 \mu \mathrm{s}$ after laser excitation. Inset: superimposed are the ions collected when a pulsed electric field is applied $50 \mu$ s after laser excitation to field ionize any ZEKE state Rydberg atoms. The figure illustrates the approximate range of excited states over which ZEKE Rydberg atoms appear to be produced. down to $n \sim 60$. Although this could mean that such states are created then rapidly are destroyed, it is more likely that this reflects a cutoff in the ZEKE state production as has been seen in other studies with both atoms and molecules [26].

In order to explore the dynamics of ultracold ZEKE states, we record the number of ZEKE state atoms as a function of time for a fixed excitation laser wavelength for a range of discrete wavelengths. Next, we record the number of ZEKE state atoms at a fixed time as the excitation laser is scanned. We discuss both experiments below.

In the first experiment, the wavelength of the second step laser is fixed to excite Rydberg levels ranging from $n=70$ to $10 \mathrm{~cm}^{-1}$ (wave numbers) above the ionization threshold. For each excitation, the delay of the pulsed electric field for the ionization and extraction is scanned (along with the ion collection gate). This allows us to map out the survival of the ZEKE states by counting the number of ZEKE Rydberg atoms that remain after a given delay time. Note that we use the term survival as opposed to lifetime because, in general, radiative decay, autoionization, black-body ionization, and collisions can each contribute-in proportions that depend on $n-$ to the destruction of the Rydberg state.

Results are shown in Fig. 5. The pulsed field and the collection gate of the photon counter are scanned with a delay ranging from 0 to $200 \mu \mathrm{s}$ in $5-\mu$ s steps and with 100 shots (at $10 \mathrm{~Hz}$ ) taken per step. The plots highlight the ZEKE data and exclude ions from autoionization and multiphoton ionization that are produced during the laser pulse and arrive at the detector around $27 \mu \mathrm{s}$. Here, the energies of the dye lasers are 200 and $250 \mu \mathrm{J} /$ pulse, respectively.

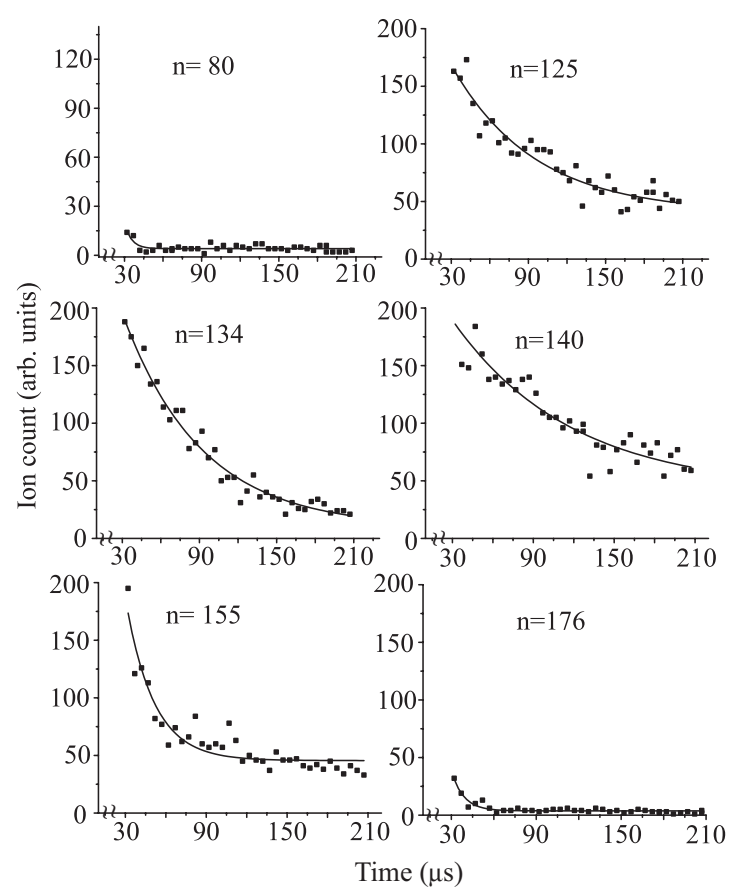

FIG. 5. Ions resulting from ZEKE Rydberg states as a function of the delay time between laser excitation and pulsed field ionization for transitions to Rydberg levels in the range of $80<n<176$ along with a simple exponential fit to the data. The fit excludes the signal before $32 \mu$ s that includes ions from prompt autoionization and multiphoton ionization. 


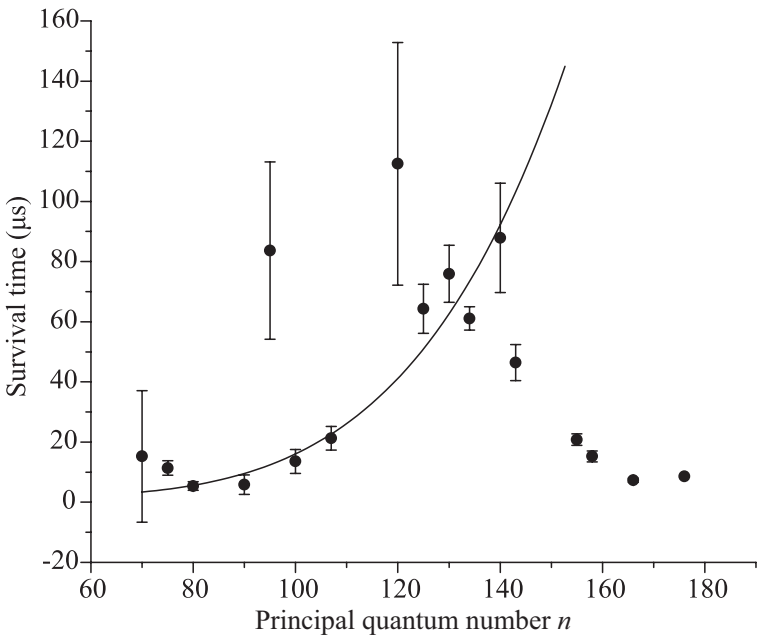

FIG. 6. Survival time as derived from the exponential fits to the data (Fig. 5) as a function of the principal quantum number $n$. The solid line is a fit to the function $\tau=a n^{b}$ for $70<n<140$ as discussed in the text.

A general trend can be observed from the graphs. Starting at $n \sim 70$, we see the onset of the creation of ZEKE Rydberg states. As $n$ is increased, the number of ZEKE atoms produced and their survival time both increase. At a wavelength corresponding to excitation near $n \sim 130$, however, the survival time starts to decrease dramatically. In an effort to provide a simple characterization of the ZEKE state survival as a function of the principal quantum number, we have fit the curve with a simple exponential. We find, in fact, that a simple exponential does a good job of characterizing the ZEKE state survival as shown in Fig. 5. Note that, for the fit, we have removed the data points before $32 \mu$ s to get rid of the contribution from the direct and fast autoionized ions.

The decay time obtained from the curve fitting is plotted against the principal quantum in Fig. 6. Since these atoms can interact with each other, in for example, a collision which destroys the Rydberg state, the decay time we measure is not necessarily the natural lifetime. Nonetheless, it is still interesting to examine how the survival time scales with $n$ and how this compares to predicted lifetimes, especially, for the part of our curve where the ZEKE survival increases with $n$. To do so, we fit our data to the function $\tau=a n^{b}$ for $70<n<140$. The points at $n=120$ and $n=95$ with the unusually large error bars were excluded from the fit. The fit yields $b=4.73 \pm 0.84$, which interestingly is in agreement with the theoretical prediction for the scaling of the natural lifetime of the high- $l$ and high- $m$ states.

A complementary way to study the ZEKE states is to fix the delay time of the PFI pulse and to scan the second excitation laser wavelength. In Fig. 7, we show ion counts as a function of laser wavelength for eight different PFI pulse delays ranging from $50 \mu \mathrm{s}$ to $1 \mathrm{~ms}$. An interesting progression of the population of ZEKE states can be observed. At 50- $\mu$ s PFI delay, the spectrum is skewed toward high- $n$ ZEKE states. At longer times, both populations decrease, as one would expect, until at the longest time recorded, the spectrum is dominated by low- $n$ ZEKE states. These data are consistent with the data previously shown. Low- $n$ ZEKE states have

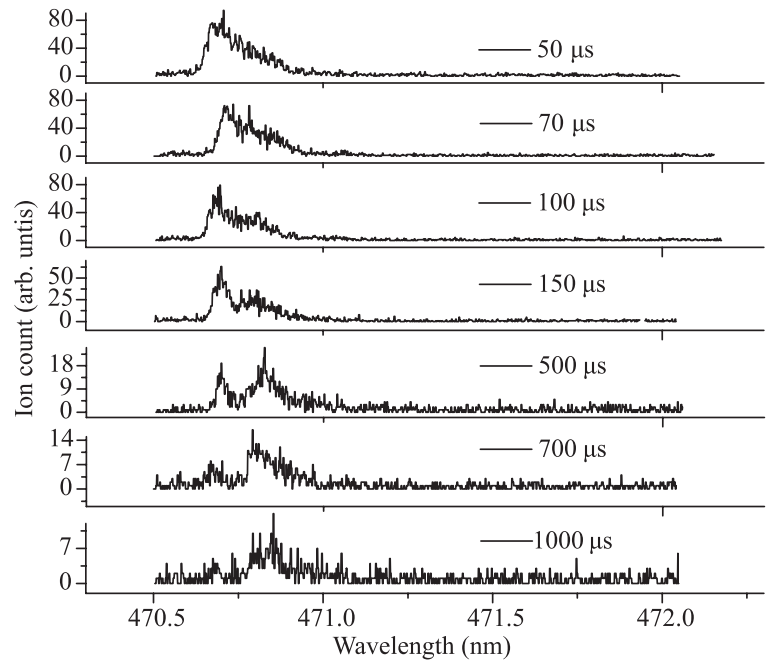

FIG. 7. Ion counts as a function of laser wavelength for eight different pulsed field ionization delays ranging from $50 \mu$ s to $1 \mathrm{~ms}$. Note that different vertical scales are used in the figure.

lower natural lifetimes but are less fragile than large high- $n$ ZEKE states that interact with the other ZEKE atoms and the environment.

We now consider several simple mechanisms that could explain the destruction of the higher- $n$ ZEKE states. These include field ionization from stray electric fields, ionization from black-body radiation, and collisions with other nearby ZEKE state Rydberg atoms. It is important to remember that these Rydberg atoms are very large with classical orbit size scaling as $n^{2}$ and are very sensitive to stray fields. We know that there is a stray electric field at the location of the MOT arising from the channel electron multiplier, although an extrapolation based on observing the collected ion signal as a function of an applied nulling voltage on one of the rings suggests the field is a factor of $\sim 3-5$ too small to ionize atoms in the $n=140$ state. Also, although the atoms are ultracold, they are still immersed in a bath of black-body radiation from the surrounding room-temperature apparatus. However, an (over)estimate of the room-temperature depopulation rate is $2.03 \times 10^{7}\left(n^{*}\right)^{-2} \mathrm{~s}^{-1}$ [37]. Depopulation from black-body redistribution is, therefore, negligible in this experiment $[38,39]$. The most likely candidate, we believe, for the decreasing survival time of high- $n$ atoms is collisions with other Rydberg atoms. Future studies will focus on clearly identifying the specific mechanisms responsible for limiting the ZEKE Rydberg state survival.

\section{CONCLUSION}

In this paper, we have reported on our investigation of the formation and survival of long-lived ZEKE Rydberg states in argon produced by pulsed laser excitation of ultracold metastable state argon atoms in a magneto-optical trap. We find that these ZEKE states are produced in a region just below the ionization limit of the ${ }^{2} P_{1 / 2}$ core Rydberg series, down to a principal quantum number of $n \sim 75$. We have characterized the survival times of these states and find that they are, indeed, orders of magnitude greater than one would find in low angular momentum states of the same $n$. For example, 
for the $d^{\prime}$ state at $n=100$, the autoionizing lifetime is 170 ps, whereas, we observe a ZEKE state survival time of $14 \mu \mathrm{s}$. Furthermore, we find that the lifetime scales as $\tau=n^{4.73 \pm 0.84}$ for states below $n \sim 130$. At this level of precision, our result cannot differentiate between an $n^{4.5}$ and an $n^{5}$ scaling but is clearly not consistent with the $n^{3}$ scaling of low- $l$ states. For higher $n$-states, the survival time decreases, likely due to the interaction between Rydberg atoms and possibly from stray fields. This work opens the door for future studies of ZEKE state dynamics in an ultracold environment. Future studies will entail control of the dynamics through the application of tailored electric fields and the investigation of the role of ZEKE states in ultracold plasma formation.

\section{ACKNOWLEDGMENT}

We wish to acknowledge the support of the Thomas Jefferson National Laboratory and Old Dominion University.
[1] T. C. Killian, S. Kulin, S. D. Bergeson, L. A. Orozco, C. Orzel, and S. L. Rolston, Phys. Rev. Lett. 83, 4776 (1999).

[2] T. C. Killian, M. J. Lim, S. Kulin, R. Dumke, S. D. Bergeson, and S. L. Rolston, Phys. Rev. Lett. 86, 3759 (2001).

[3] T. F. Gallagher, P. Pillet, M. P. Robinson, B. Laburthe-Tolra, and M. Noel, J. Opt. Soc. Am. B 20, 1091 (2003).

[4] S. D. Bergeson and F. Robicheaux, Phys. Rev. Lett. 101, 073202 (2008).

[5] G. Reiser, W. Habenicht, K. Muller-Dethlefs, and E. W. Schlag, Chem. Phys. Lett. 152, 119 (1988).

[6] M. C. R. Cockett, Chem. Soc. Rev. 34, 935 (2005).

[7] A. Held and E. W. Schlag, Acc. Chem. Res. 31, 467 (1998).

[8] R. F. Stebbings and F. B. Dunning, Phys. Rev. A 8, 665 (1973).

[9] F. B. Dunning and R. F. Stebbings, Phys. Rev. A 9, 2378 (1974).

[10] C. Delsart and J.-C. Keller, Phys. Rev. A 28, 845 (1983).

[11] J. Landais, M. Huet, H. Kucal, and T. Dohnalik, J. Phys. B 28, 2395 (1995).

[12] S. M. Koeckhoven, W. J. Buma, and C. A. de Lange, Phys. Rev. A 51, 1097 (1995).

[13] U. Hollenstein, H. Palm, and F. Merkt, Rev. Sci. Instrum. 71, 4023 (2000).

[14] Y.-Y. Lee, T.-Y. Dung, R.-M. Hsieh, J.-Y. Yu, Y.-F. Song, G. H. Ho, T.-P. Huang, W.-C. Pan, I.-C. Chen, S.-Y. Tu, A. H. Kung, and L. C. Lee, Phys. Rev. A 78, 022509 (2008).

[15] M. A. Baig, S. Mahmood, R. Mumtaz, M. Rafiq, M. A. Kalyar, S. Hussain, and R. Ali, Phys. Rev. A 78, 032524 (2008).

[16] D. Klar, K. Harth, J. Ganz, T. Kraft, M.-W. Ruf, H. Hotop, V. Tsemekhman, K. Tsemekhman, and M. Amusia, Z. Phys. D 23, 101 (1992)

[17] J. M. Weber, K. Ueda, D. Klar, J. Kreil, M.-W. Ruf, and H. Hotop, J. Phys. B 32, 2381 (1999).

[18] I. D. Petrov, V. L. Sukhorukov, and H. Hotop, J. Phys. B 35, 323 (2002).
[19] I. D. Petrov, V. L. Sukhorukov, and H. Hotop, J. Phys. B 36, 119 (2003).

[20] J. D. Wright, T. J. Morgan, L. Li, Q. Gu, J. L. Knee, I. D. Petrov, V. L. Sukhorukov, and H. Hotop, Phys. Rev. A 77, 062512 (2008).

[21] V. L. Sukhorukov, I. D. Petrov, M. Schäfer, F. Merkt, M.-W. Ruf, and H. Hotop, J. Phys. B 45, 092001 (2012).

[22] L. E. Wright, E. L. Snow, S. R. Lundeen, and W. G. Sturrus, Phys. Rev. A 75, 022503 (2007).

[23] M. E. Hanni, J. A. Keele, S. R. Lundeen, and W. G. Sturrus, Phys. Rev. A 78, 062510 (2008).

[24] A. Held, U. Aigner, L. Y. Baranov, H. L. Selzle, and E. W. Schlag, Chem. Phys. Lett. 299, 110 (1999).

[25] J. D. D. Martin, J. W. Hepburn, and C. Alcaraz, J. Phys. Chem. A 101, 6728 (1997).

[26] A. Muhlpfordt and U. Even, J. Chem. Phys. 103, 4427 (1995).

[27] F. Merkt, J. Chem. Phys. 100, 2623 (1994).

[28] M. J. J. Vrakking and Y. T. Lee, J. Chem. Phys. 102, 8833 (1995).

[29] W. Chupka, J. Chem. Phys. 98, 4520 (1993).

[30] T. P. Softley, A. J. Hudson, and R. Watson, J. Chem. Phys. 106, 1041 (1997).

[31] M. Bixon and J. Jortner, J. Chem. Phys. 103, 4431 (1995).

[32] M. J. J. Vrakking, J. Phys. Chem. A 101, 6761 (1997).

[33] F. Merkt and R. N. Zare, J. Chem. Phys. 101, 3495 (1994).

[34] T. Gallagher, Rydberg Atoms (Cambridge University Press, New York, 1994).

[35] S. K. Dutta, D. Feldbaum, A. Walz-Flannigan, J. R. Guest, and G. Raithel, Phys. Rev. Lett. 86, 3993 (2001).

[36] C. I. Sukenik and H. C. Busch, Phys. Rev. A 66, 051402 (2002).

[37] J. W. Farley and W. H. Wing, Phys. Rev. A 23, 2397 (1981).

[38] W. E. Cooke and T. F. Gallagher, Phys. Rev. A 21, 588 (1980).

[39] I. I. Beterov, I. I. Ryabtsev, D. B. Tretyakov, and V. M. Entin, Phys. Rev. A 79, 052504 (2009). 